\title{
Análise das condições para a criação de uma rede entre órgãos públicos para regulação do setor de saúde suplementar
}

\author{
Analysis of the Conditions for the Creation of a Network between Public \\ Agencies in the Supplemental Health Sector Regulation
}

\author{
André Luis Pereira Duarte ${ }^{1}$ \\ Peter Bent Hansen ${ }^{2}$ \\ Grace Vieira Becker ${ }^{3}$ \\ Jane Lucia Santos ${ }^{4}$
}

\begin{abstract}
Resumo
As estruturas em rede, no âmbito do setor público, apresentam uma expansão na atualidade, produzindo uma nova realidade administrativa. No contexto do setor público brasileiro, ainda é um tema pouco explorado. A instituição pública responsável por atuar na regulação de saúde no Brasil é a Agência Nacional de Saúde Suplementar (ANS). Outros órgãos públicos que interagem nesse processo incluem o Programa de Orientação e Proteção do Consumidor (PROCON), Ministério Público, Defensoria Pública e Poder Judiciário, porém as atividades desses órgãos não estão integradas. $O$ objetivo do presente estudo é analisar as condições para a criação de uma rede entre os órgãos públicos que participam do processo regulatório de saúde suplementar no Rio Grande do Sul. Para tanto, foi realizada uma pesquisa qualitativa, exploratória, adotando-se como estratégia um estudo de caso. Os dados foram coletados por meio de entrevistas em profundidade com gestores dos órgãos públicos envolvidos na regulação da saúde suplementar. A análise foi realizada com base em nove categorias propostas como condições necessárias para a criação da rede - receptividade, estímulo, confiança, cooperação, suporte político, suporte técnico operacional, organização articuladora, instrumentos de coordenação, e interação constante e duradoura. Os resultados da pesquisa apontam que os entrevistados vislumbram possibilidades de ganhos efetivos a partir da atuação em rede, incluindo maior efetividade da regulação da saúde suplementar. Todavia eles não possuem muita clareza a respeito dos aspectos que envolvem a atuação em rede, sendo necessária melhor compreensão sobre o tema. Constata-se, assim, um campo de pesquisa a ser mais explorado.
\end{abstract}

Palavras-chave: Redes. Relações interorganizacionais. Setor Público. Saúde Suplementar.

\begin{abstract}
Network structures within the public sector have an expansion at present, producing a new administrative reality. In the context of the Brazilian public sector, it is still a relatively unexplored subject. The public institution responsible for proceed health regulation in Brazil is the National Agency of Supplemental Health (ANS). Other government agencies that interact in this process include the Orientation Program and Consumer Protection (OPCP), Public Ministry, Public Defender's Office and the Judiciary. However, the activities of these agencies are not integrated. The aim of this study is to analyze the conditions for the creation of a network between public agencies that participate in the regulatory process of supplemental health in Rio Grande do Sul. For this, a qualitative, exploratory research was conducted, adopting a case study strategy. Data were collected through interviews with managers of public agencies involved in the regulation of the supplemental health. The analysis was based on nine categories proposed as necessary conditions for the creation of the network - receptivity, stimulation, trust, cooperation, political support, operational technical support, articulated organization, coordination instruments, and constant and lasting
\end{abstract}

Brasil. Afiliação: PPGAd / PUCRS - Pontifícia Universidade Católica do Rio Grande do Sul. Email: alp-duarte@yahoo.com.br

Possui mestrado e doutorado em Engenharia de Produção pela Universidade Federal do Rio Grande do Sul (2004). Atualmente é professor e pesquisador do Programa de Pós-Graduação em Administração - PPGAd, da Pontifícia Universidade Católica do Rio Grande do Sul - PUCRS, além de professor nas Faculdades de Administração e Engenharia de Produção da PUCRS. Brasil. Afiliação: UFRGS - Universidade Federal do Rio Grande do Sul. Lattes: http://lattes.cnpq.br/6199838733654819. Email: hansen.peter.57@gmail.com

3 Possui doutorado em Administração pela Universidade de São Paulo e Pós Doutorado pela Université du Québec à Montréal, no Canadá. Brasil. Afiliação: USP - Universidade de São Paulo. Lattes: http://lattes.cnpq.br/1311749423678495. Email: gbecker@usp.br

4 Possui mestrado e doutorado em Engenharia e Gestão do Conhecimento pela Universidade Federal de Santa Catarina (PPEGC/UFSC) - com atuação como pesquisadora do Núcleo de Gestão para Sustentabilidade - NGS. Brasil. Afiliação: PUCRS - Pontifícia Universidade Católica do Rio Grande do Sul. Lattes: http://lattes.cnpq.br/3200475850528094. Email: janejlss@gmail.com 
interaction. The survey results show that respondents envisage effective possibilities of earnings from network operations, including greater effectiveness of the supplemental health regulation. However, they do not have much distinctness about the aspects that involve working in networks, which requires better understanding of the subject. Therefore, it is a research field that can be further explored.

Keywords: Networks. Interorganizational Relations. Public Sector. Supplemental Health.

\section{Introdução}

As estruturas em rede, no âmbito do setor público, apresentam um aumento significativo nas últimas décadas, ocasionando uma nova realidade administrativa em virtude dos relacionamentos estabelecidos entre Estado e sociedade e entre organizações nas esferas de governo (LOIOLA; MOURA, 1996; FLEURY; OUVERNEY, 2007). Essas novas formas surgem devido ao esgotamento da capacidade de integração das instituições representativas tradicionais, além da ineficácia das organizações burocráticas e do modelo de planejamento global e centralizado (MOURA, 1998) e também da necessidade de participação pública no setor da saúde, por exemplo, enquanto elemento estruturante das políticas públicas (CRISÓSTOMO; SANTOS, 2018).

No Brasil, as experiências do Estado com as relações interorganizacionais são recentes. Como exemplo tem-se o modelo de parcerias público-privadas (PPPs), por meio da criação da Lei $n^{\circ} 11.079 / 2004$, constituindose num novo marco na legislação brasileira como espaço de cooperação entre a administração pública e o setor privado. Além disso, as redes têm sido utilizadas no setor público brasileiro para a implementação de políticas públicas, tais como alfabetização de jovens e adultos, regionalização da saúde, combate à fome e erradicação do trabalho infantil (BARBOSA; MEDEIROS, 2002; FLEURY; OVERNEY, 2007; FLEURY; MIGUELETO; BLOCH, 2002; AMANTINO-DE-ANDRADE, 2005).

Nesse sentido, as políticas regulatórias se caracterizam como uma área propícia para a atuação em rede, como é o caso do setor de saúde suplementar, que pode se organizar das mais variadas formas e promover relacionamentos nos mais diversos campos, tornando as possibilidades de regulação extremamente diversificadas (SANTOS, 2006). A instituição pública responsável por atuar diretamente na regulação do setor é a Agência Nacional de Saúde Suplementar (ANS), sendo que, nesse processo, também interagem outros órgãos públicos, tais como o Programa de Orientação e Proteção ao Consumidor (PROCON), Ministério Público, Defensoria Pública e Poder Judiciário, entre outros (GIOVANELLA; RIBEIRO; COSTA, 2002; LIMA; LIMA, 2010). Porém não há uma integração da atuação desses órgãos, sendo comum a desinformação, a ausência de mecanismos de coordenação e a superposição de funções (PECI; COSTA, 2002; LAZZARINI, 2009).

Diante das dificuldades e dos desafios enfrentados para a criação e manutenção das redes no âmbito da administração pública (FLEURY; OUVERNEY, 2007), inclusive no âmbito da saúde pública (PETERS et al., 2017), este estudo tem por objetivo identificar e analisar as condições necessárias para criação de uma rede entre os órgãos públicos que participam - direta ou indiretamente - do processo regulatório do setor de saúde suplementar do Rio Grande do Sul, Brasil. Nesse sentido, busca-se estudar se as redes interorganizacionais, aplicadas ao contexto dos órgãos públicos, podem ser uma boa estratégia para uma maior efetividade dos serviços públicos (FLEURY; OUVERNEY, 2007).

Além desta introdução, o artigo está estruturado em mais cinco seções. Na próxima seção é abordado o referencial teórico. Na terceira seção é apresentado o contexto de realização desta pesquisa, isto é, o cenário da regulação dos serviços de saúde. Na seção 4 é feita a descrição dos procedimentos metodológicos. Na seção 5 são apresentados os principais resultados. Por fim, na seção 6 , são incluídas as considerações finais, seguidas das referências utilizadas.

\section{Redes interorganizacionais e administração pública}

Nesta seção são discutidos os tópicos que embasam a presente pesquisa, a saber: as características das redes interorganizacionais, as condições necessárias para a criação destas e a abordagem das redes na administração pública no Brasil. 


\subsection{Condições necessárias para a formação de redes inteorganizacionais}

As redes são criadas em diferentes configurações, em contextos distintos e a partir de múltiplas expressões culturais (CASTELLS, 1999a), e promovem vários benefícios estratégicos para as organizações participantes, tais como aprendizagem, compartilhamento de informações, geração e transmissão de conhecimento, entre outros (TURETA; LIMA, 2011). Nesse sentido, não existirão duas redes interorganizacionais estruturadas da mesma forma, devido às muitas possibilidades para a sua criação (BALESTRIN; VERSCHOORE, 2008), aos múltiplos interesses estratégicos (TURETA; LIMA, 2011) e aos diferentes fatores antecedentes (CHEN, 2010; GULATI; WOHLGEZOGEN; ZHELYAZKOV, 2012), os quais são condições necessárias para a formação de redes.

As atuais discussões teóricas da área indicam algumas direções para se entender as condições necessárias para a criação das redes, no entanto ainda não há consenso ou uma resposta plena para essa questão (BALESTRIN; VERSCHOORE, 2008). Vários pesquisadores (p.ex. OLIVER, 1990; BALESTRIN; VARGAS, 2002; FLEURY; OUVERNEY, 2007; ALBANO: DE ARAUJO; REINHARD, 2017) concordam, todavia, que o desempenho e o sucesso das redes dependerão de diversos atributos essenciais para a sua formação. No Quadro 1 estão sintetizadas os principais fatores (condições) para criação de redes identificados na literatura sobre o tema.

\section{Quadro 1 - Condições necessárias para a criação das redes interorganizacionais}

\begin{tabular}{|c|c|c|}
\hline $\begin{array}{l}\text { Condições } \\
\text { Necessárias }\end{array}$ & Fatores relacionados & Autores \\
\hline $\begin{array}{l}\text { Objetivos } \\
\text { comuns }\end{array}$ & $\begin{array}{l}\text { Necessidades: criar elos ou trocas com outras organizações; } \\
\text { Reciprocidade: intercâmbio de idéias, desenvolvimento de visão } \\
\text { estratégica, definição da contribuição dos parceiros, aceitação de } \\
\text { que o parceiro é uma organização semelhante; } \\
\text { Eficiência: melhorar o desempenho organizacional e buscar uma } \\
\text { forma de minimizar os custos transacionais; } \\
\text { Estabilidade: fortificar o padrão de interdependência; } \\
\text { Redução de riscos e incertezas: falta de recursos e conhecimento; } \\
\text { Legitimidade: melhorar a reputação, o prestígio e o acordo com as } \\
\text { normas que prevalecem no ambiente institucional; } \\
\text { Interesse em cooperação: busca de consensos, processos de } \\
\text { negociação, geração de maior comprometimento dos atores. }\end{array}$ & $\begin{array}{l}\text { Oliver (1990); Castells } \\
\text { (1999a); Marcon e } \\
\text { Moinet (2001); Balestrin } \\
\text { e Vargas (2002); Keast } \\
\text { et al. (2004); Nakano } \\
\text { (2005); Agranoff (2006); } \\
\text { Fleury e Ouverney } \\
\text { (2007); Balestrin e } \\
\text { Verschoore (2008). }\end{array}$ \\
\hline Receptividade & $\begin{array}{l}\text { - Aceitação: os atores devem estar receptivos à lógica da rede; } \\
\text { - Abandono do individualismo; } \\
\text { - Grau de compatibilidade e congruência de valores. }\end{array}$ & \begin{tabular}{|l} 
Marcon e Moinet \\
(2001); Fleury e \\
Ouverney (2007).
\end{tabular} \\
\hline Confiança & $\begin{array}{l}\text { Cumplicidade: atores que não desenvolvam concorrência; } \\
\text { Confiança construída desde o período de formação da rede; } \\
\text { Confiança interpessoal: confiança entre pessoas; } \\
\text { Confiança institucional: confiança em agentes impessoais, como } \\
\text { as instituições sociais, políticas e econômicas. }\end{array}$ & $\begin{array}{l}\text { Costa (2000); Marcon } \\
\text { e Moinet (2001); } \\
\text { Lagemann (2004). }\end{array}$ \\
\hline $\begin{array}{l}\text { Interação } \\
\text { constante }\end{array}$ & $\begin{array}{l}\text { Comunicação: intensidade e frequência; } \\
\text { Contatos: virtuais e face a face; } \\
\text { Infraestrutura: TICs, local, material, etc.; } \\
\text { Aspectos sociais: instrumentos de integração (entre os } \\
\text { funcionários das organizações). }\end{array}$ & $\begin{array}{l}\text { Castells (1999a); Hage } \\
\text { e Hollingsworth (2000); } \\
\text { Marcon e Moinet } \\
\text { (2001); Balestrin e } \\
\text { Verschoore (2008). } \\
\end{array}$ \\
\hline $\begin{array}{l}\text { Coordenação } \\
\text { dos atores e } \\
\text { procedimentos }\end{array}$ & $\begin{array}{l}\text { - Estabelecimento de áreas de atuação; } \\
\text { - Análise conjunta dos problemas, soluções em comum; } \\
\text { - Instrumentos contratuais: normas, procedimentos, conjunto de } \\
\text { regras e ética entre os participantes; } \\
\text { - Instrumentos estratégicos: planos estratégicos, planos de ação; } \\
\text { - Instrumentos de tomada de decisão: reuniões dos atores; } \\
\text { - Organização articuladora: animador, integrador de esforços. }\end{array}$ & $\begin{array}{l}\text { Grandori e Soda (1995) } \\
\text { Agranoff e McGuire } \\
\text { (1999); Peci e Costa } \\
\text { (2002) e Balestrin e } \\
\text { Verschoore (2008). }\end{array}$ \\
\hline
\end{tabular}

Fonte: Elaborado pelos autores. 
Apresentadas as condições relevantes para a criação de redes interorganizacionais, passa-se a abordar essas redes no ambiente público e suas características.

\subsection{Redes na administração pública}

O fenômeno da criação de redes na administração pública tem sido um tema discutido desde a década de 1990 (FLEURY; OUVERNEY, 2007). Entretanto, de acordo com Fleury e Ouverney (2007, p. 41), antes dos anos 1990 a temática já era tratada indiretamente na literatura internacional quando se estudava "o desenvolvimento de estratégias conjuntas entre atores governamentais, privados e organizações da sociedade social". Assim, ainda é um tema que merece atenção e necessita de estudos empíricos (PROVAN; LEMAIRE, 2012; NING, 2013).

$\mathrm{Na}$ área pública, uma estrutura em rede é constituída por uma colaboração ativa e organizada de organizações públicas, privadas e sem fins lucrativos, destinadas a alcançar um ou mais objetivos estabelecidos. Conceitualmente, pode ser vista como um modelo de gestão de políticas através de estruturas interdependentes que envolvem múltiplas organizações (O'TOOLE, 1997; PROVAN; LEMAIRE, 2012).

No campo de atuação do Estado, as redes são entendidas como formas de articulação entre agências governamentais e/ou entre elas e redes sociais, organizações privadas e outros grupos, para enfrentar problemas sociais e implementar políticas públicas (LOIOLA; MOURA, 1996; PROVAN; LEMAIRE, 2012). Estruturam-se, portanto, novas formas de laços entre Estado e sociedade e entre agências e esferas de governo.

Além disso, para Walker et al. (2007), existem dois tipos mais significativos de redes na administração pública: as redes de entrega de serviços e as redes de políticas. Goldsmith e Eggers (2006) abordam as redes de entrega de serviços como um novo modelo denominado "governar em rede", no qual as instituições públicas dependem menos de servidores públicos em papéis tradicionais e mais de uma teia de parcerias, contratos e alianças para realizar a entrega de serviços públicos. As políticas públicas, depois de formuladas, desdobram-se em planos, programas, projetos, bases de dados ou sistemas de informação e pesquisas (SOUZA, 2006). Sendo assim, por meio das redes de políticas, é possível obter os recursos dispersos e dar uma resposta mais eficiente e eficaz aos problemas de políticas públicas (FLEURY, 2005). Elas surgem a partir do crescimento da interdependência funcional entre atores públicos e privados na implementação de políticas públicas (FLEURY, 2005; PROVAN; LEMAIRE, 2012).

Dentre os principais atributos das redes de políticas, a interdependência representa um elemento fundamental para o surgimento desses arranjos (PECl; COSTA, 2002; OUVERNEY, 2005). Assim, a interdependência é o principal fator que influencia a coordenação interorganizacional, sendo fundamental para o processo de institucionalização da rede, uma vez que sua estrutura é criada a partir dos fatores que impulsionam essa interdependência (FLEURY; OUVERNEY, 2007). Porém, tendo em vista as características das redes de políticas, Fleury e Ouverney (2007) apontam tanto vantagens como desvantagens relativas a essas redes. Neste sentido, ressaltam-se alguns déficits de eficácia que as redes de políticas sociais podem apresentar, tais como a falta de articulação das políticas econômicas e sociais, a tendência a excluir os temas conflitivos e concentrar-se em questões administrativas de menor importância, a necessidade de elaboração de planejamentos estratégicos e do gestor público assumir o papel de um negociador e deixar de ser um mero cumpridor de planos (FLEURY; OUVERNEY, 2007).

Nesse contexto, verifica-se a existência de algumas questões específicas às redes na administração pública, tais como: 1) o estímulo para a criação da rede: verificação da existência de uma necessidade (informação, conhecimento, melhoria da eficiência e da prestação dos serviços) para as trocas entre as organizações, de forma que seja possível identificar as interdependências existentes, tornando viável a definição da finalidade e dos objetivos comuns da rede (FLEURY, 2005; AGRANOFF, 2006; FLEURY; OUVERNEY, 2007; BALESTRIN; VERSCHOORE, 2008); e 2) o estabelecimento de um suporte institucional para sustentar a institucionalização e o desenvolvimento dos padrões de interdependência existentes entre os atores que compõem a rede (OUVERNEY, 2005; OUVERNEY; FLEURY, 2006; FLEURY; OUVERNEY, 2007), uma vez que, muitas vezes, pertencem a poderes diferentes (por exemplo, executivo e judiciário). Esse suporte institucional dependerá, de um lado, de um suporte político no nível do cluster de atores e recursos 
(desenvolver estratégias de compartilhamento decisório), inclusão de interesses plurais (formular estratégias para ampliar a representatividade, construir consensos e projetos hegemônicos, evitar deserções em momentos cruciais), e redução das assimetrias de poder (evitar bloqueios e impasses, de forma que o processo de negociação e geração de consensos possa ser ágil, com a solução rápida de problemas que requerem ação imediata) (FLEURY; OUVERNEY, 2007). Dependerá, também, de um suporte técnico-operacional, destinado a garantir a articulação e integração das bases de recursos e de competências, com o objetivo de promover a racionalidade sistêmica na alocação de recursos, garantir a flexibilidade operacional e criar elementos de suporte decisório (FLEURY; OUVERNEY, 2007). Entretanto ainda é um desafio a "institucionalização de mecanismos participativos e de espaços regulares e efetivos de participação pública dos cidadãos e seus representantes no contexto de saúde, enquanto parceiros-chave na mudança e corresponsáveis nos processos de decisão em saúde" (CRISÓSTOMO; SANTOS, 2018, p. 179).

Na próxima seção deste trabalho é descrito o ambiente específico de análise neste estudo, que se refere ao setor de saúde pública suplementar.

\section{Setor de saúde suplementar no Brasil e no Rio Grande do Sul}

A expansão do setor de saúde suplementar, surgido na década de 1930 no mercado brasileiro, iniciouse na década de 60, por meio das empresas médicas (medicina de grupo) que eram financiadas por meio de convênios com a Previdência Social (BAHIA, 2001; MACERA; SAINTIVE, 2004; FIGUEIREDO, 2006). Na década de 1980, a partir da Constituição Brasileira de 1988, além dos serviços públicos de saúde, o Poder Público admitiu que as ações e serviços de saúde também pudessem ser prestados pela iniciativa privada. Nos anos 1990, houve um forte incremento das taxas de crescimento dos planos e seguros de saúde privados, com a perspectiva de aumento do poder de compra de expressivo contingente populacional (BAHIA, 2001; FIGUEIREDO, 2006).

Por outro lado, destacam-se algumas dificuldades decorrentes de determinados comportamentos nesse setor, tanto dos consumidores quanto das empresas, tais como: a) risco moral; b) seleção adversa; e c) seleção de riscos (BAHIA, 2001; MACERA; SAINTIVE, 2004; FIGUEIREDO, 2006; CECHIN, 2008). O risco moral refere-se à mudança de comportamento do consumidor, com o aumento da utilização de serviços, em função de não ter que suportar o custo total do atendimento. Já a seleção adversa, consiste na autoexclusão dos consumidores que têm gastos com saúde esperados inferiores ao valor cobrado no mercado. A seleção de risco, por sua vez, surge quando, para protegerem-se contra a combinação de baixos prêmios e alto potencial de custo, as empresas criam barreiras à entrada dos segurados no sistema, excluindo os de alto risco, como os idosos.

Nesse contexto, diante da complexidade do mercado e de outros pontos, houve consenso em torno da necessidade de intervenção estatal e da criação de um marco legal para sua regulamentação (BAHIA; VIANA, 2002; FIGUEIREDO, 2006). Dessa forma, a partir de 1998, com a aprovação da Lei n. ${ }^{\circ} 9.656$, a regulação dos planos de saúde começou a ocorrer no Brasil.

A regulação pode ser entendida como a capacidade de intervir nos processos de prestação de serviços, alterando ou orientando a sua execução, por meio de mecanismos indutórios, normatizadores, regulamentadores ou restritores (SANTOS, 2006). Desse modo, a Agência Nacional de Saúde Suplementar (ANS) é o órgão responsável pela regulação, normatização, controle e fiscalização das atividades relativas à assistência suplementar à saúde.

No Quadro 2 são apresentadas as principais características da regulação do setor de saúde suplementar no Brasil e os principais agentes desse mercado. 


\section{Quadro 2 - Características da regulação do setor de saúde suplementar no Brasil}

\begin{tabular}{|l|l|}
\hline Característica & Descrição \\
\hline Tamanho do mercado & $\begin{array}{l}\text { Cerca de } 50 \text { milhões de clientes, mais de duas mil operadoras e mais de setenta mil } \\
\text { estabelecimentos de saúde atuando no setor. }\end{array}$ \\
\hline Órgão regulador & $\begin{array}{l}\text { Agência Nacional de Saúde suplementar (ANS): agência reguladora vinculada } \\
\text { ao Ministério da Saúde, responsável pela regulação, normatização, controle e } \\
\text { fiscalização das atividades. }\end{array}$ \\
\hline Agentes do mercado & $\begin{array}{l}\text { Os clientes (beneficiários); Os prestadores ou provedores de serviço (hospitais, } \\
\text { médicos, clínicas e laboratórios); As operadoras de planos e seguros-saúde; O } \\
\text { órgão regulador; e A indústria de equipamentos, materiais e fármacos. }\end{array}$ \\
\hline $\begin{array}{l}\text { Tipos de operadoras de } \\
\text { saúde no Brasil }\end{array}$ & $\begin{array}{l}\text { Administradoras; Medicinas/odontologias de grupo; Cooperativas Médicas/ } \\
\text { odontológicas; Filantropias; Autogestões; Seguros-saúde. }\end{array}$ \\
\hline Objetivos da Regulação & $\begin{array}{l}\text { Corrigir eventuais falhas de mercado em qualquer uma das interaços: clientes/ } \\
\text { operadoras, operadoras/prestadores e prestadores/clientes, mantendo a } \\
\text { estabilidade do mercado, o controle da assimetria das informações e a maximização } \\
\text { da participação do consumidor. }\end{array}$ \\
\hline
\end{tabular}

Fonte: Elaborado pelos autores.

No mercado de saúde suplementar do estado do Rio Grande do Sul/RS, a ANS desenvolve seu processo regulatório por meio da fiscalização, a qual é efetivada por seu núcleo regional, localizado na cidade de Porto Alegre. Esse mercado é composto por cerca de 2,3 milhões de clientes, mais de 500 operadoras em atividade e mais de 6.000 estabelecimentos de saúde que atendem planos privados de saúde (BRASIL, 2010).

A articulação com os órgãos de defesa do consumidor é uma atividade que compete à Diretoria de Fiscalização da ANS e ao seu núcleo regional no estado do RS. Essa articulação tem como objetivo realizar a proteção e defesa do consumidor de serviços privados de assistência à saúde (FIGUEIREDO, 2006) e evitar conflitos e a duplicidade de regulações (CUNHA, 2003). Nesse processo, a ANS, no estado do RS, pode interagir com diversos órgãos públicos que se envolvem na regulação do mercado de saúde suplementar, tais como Programa de Orientação e Proteção ao Consumidor (PROCON), estadual e municipal, Ministério Público (estadual e federal), Defensoria Pública Estadual e Poder Judiciário.

No Quadro 3 são apresentados os órgãos públicos envolvidos no processo de regulação do mercado de saúde suplementar no Brasil e sua respectivas atuações.

Quadro 3 - Os órgãos públicos envolvidos no processo de regulação do mercado de saúde suplementar

\begin{tabular}{|l|l|}
\hline Órgão & Atuação \\
\hline \multirow{3}{*}{ ANS } & $\begin{array}{l}\text { - A regulação do setor no estado do RS: a fiscalização (direta ou reativa e indireta ou pró- } \\
\text { ativa) é a ação central; } \\
\text { - Estrutura: apenas um Núcleo regional, vinculado à Diretoria de Fiscalização (órgão da ANS } \\
\text { encarregado de representar os interesses dos consumidores dos planos de saúde). }\end{array}$ \\
\hline PROCON's & $\begin{array}{l}\text { - Atuação: orientar, proteger e defender os direitos e interesses dos consumidores; } \\
\text { - Organismos autônomos: não estão submetidos a uma hierarquia e têm a atribuição } \\
\text { precípua de zelar pela aplicação do CDC; } \\
\text { - Foco no consumidor: avaliar a qualidade dos produtos e serviços prestados; } \\
\text { - Penalidades administrativas: no que se refere aos planos de saúde, aplicar multas e } \\
\text { solicitar a verificação da situação das operadoras junto à ANS. }\end{array}$ \\
\hline Ministério & $\begin{array}{l}\text { - Atuação: defesa da sociedade e dos interesses sociais e individuais indisponíveis, } \\
\text { promovendo a defesa do consumidor, em nível judicial; } \\
\text { Público } \\
\text { - Competências: i) promover ação penal contra aqueles que praticam condutas típicas } \\
\text { descritas no CDC; ii) mover ação para obter declaração de nulidade de cláusula contratual } \\
\text { estabelecida em detrimento do consumidor; iii) instaurar e presidir inquérito civil; e, iv) fazer } \\
\text { recomendações para que órgãos da administração indireta (no caso a ANS) tenham maior } \\
\text { celeridade e racionalizem os procedimentos administrativos; } \\
\text { - Promotorias especializadas com atribuições de proteção ao consumidor: atuam } \\
\text { na defesa coletiva do consumidor, em prol de interesses difusos, coletivos e individuais } \\
\text { homogêneos dotados de relevância social (ex: casos envolvendo planos de saúde). }\end{array}$ \\
\hline
\end{tabular}


Quadro 3 - Os órgãos públicos envolvidos no processo de regulação do mercado de saúde suplementar (Continuação)

\begin{tabular}{|l|l|}
\hline Órgão & Atuação \\
\hline Defensoria & $\begin{array}{l}\text { - Atuação: orientação jurídica, judicial e extrajudicial e a defesa, em todos os graus, dos } \\
\text { necessitados; } \\
\text { - Competências: patrocínio dos direitos e interesses do consumidor, da criança e do } \\
\text { adolescente, do idoso e dos deficientes físicos; } \\
\text { - Direitos coletivos que cabem à Defensoria Pública tutelar: i) direitos do consumidor; e ii) } \\
\text { direitos coletivos do idoso (aumentos abusivos dos planos de saúde dos idosos). }\end{array}$ \\
\hline $\begin{array}{l}\text { Poder } \\
\text { Judiciário }\end{array}$ & $\begin{array}{l}\text { - Atuação: interpretação do direito individual e coletivo conforme a legislação em vigor; } \\
\text { - Sobreposição do seu papel: liberação de tratamentos médicos não previstos e, em alguns } \\
\text { - Judicialização da política: atua cada vez mais na definição de políticas públicas, seja } \\
\text { porque o Legislativo não foi capaz de editar leis precisas e claras, seja porque o Executivo } \\
\text { foi incapaz de operacionalizar de forma adequada essas políticas. }\end{array}$ \\
\hline
\end{tabular}

Fonte: Elaborado pelos autores.

Conforme visto, o processo de regulação da saúde suplementar no RS pressupõe uma forte intervenção do Estado, com a participação de diferentes órgãos públicos. Entretanto não há uma integração da atuação desses órgãos e de suas interfaces nas ações fiscalizatórias, sendo comum a desinformação, a ausência de mecanismos de coordenação e a sobreposição de funções (LAZZARINI, 2009).

A seguir, na próxima seção, são descritos os procedimentos metodológicos adotados para realizar o estudo empírico apresentado neste artigo.

\section{Procedimentos metodológicos}

O estudo caracteriza-se como uma pesquisa qualitativa, exploratória, de corte transversal, realizado em um estudo de caso único incorporado. A unidade de análise considerada foi o processo regulatório do setor de saúde suplementar, que envolve as instituições públicas com sede no Rio Grande do Sul-RS. Essas instituições públicas, direta ou indiretamente, participam do processo. Tal escolha justifica-se porque as interconexões entre os atores de uma rede são a parte elementar do fenômeno estudado (COLLIS; HUSSEY, 2005).

Em relação à coleta de dados, foram realizadas entrevistas em profundidade, semiestruturadas, cujos sujeitos foram os gestores públicos das instituições já caracterizadas neste estudo (seção 3). Antes de se iniciar a coleta de dados, o instrumento de pesquisa preliminar foi submetido à validação de face e conteúdo por dois especialistas (profissionais da área de métodos de pesquisa e gestão de redes interorganizacionais). Em seguida, foi realizado o pré-teste com o objetivo de ajustar o roteiro de entrevistas e validar o instrumento.

A partir de então, foram realizadas dezesseis entrevistas, sendo 3 representantes da ANS, 3 do PROCON, 4 do Ministério Público, 3 da Defensoria Pública e 3 do Poder Judiciário, durante os meses de dezembro de 2012 e fevereiro de 2013. Vale ressaltar que a seleção dos entrevistados obedeceu aos seguintes critérios: (i) ser servidor de um dos órgãos participantes da pesquisa; (ii) atuar no setor de saúde suplementar e/ou de defesa do consumidor; e (iii) ter experiência de, no mínimo, três anos na área de atuação. Os dados coletados por meio das entrevistas gravadas foram transcritos e analisados com base na técnica de categorização, conforme Bardin (2004), totalizando aproximadamente 16 horas de gravação (com duração média de uma hora para cada entrevista) e 240 páginas de transcrição.

Para o desenvolvimento do instrumento de pesquisa, o roteiro de entrevistas, foi desenvolvida uma estrutura de análise, tendo como base a contextualização do setor (seção 3) e o referencial teórico sobre redes interorganizacionais e redes na administração pública (seção 2). Assim, as análises foram realizadas tendo como foco nove categorias agrupadas nas seguintes dimensões:

1 - Ambiente favorável: questões relacionadas com a lógica de atuação em rede. Composta por quatro categorias: a) Receptividade: aceitação por parte dos órgãos públicos para atuar em rede, com tolerância a 
sua lógica e eliminação de "egos" individuais e institucionais (PECl; COSTA, 2002); b) Estímulo: Definição da finalidade (MARCON; MOINET, 2001), dos objetivos comuns da rede (CASTELLS, 1999; BALESTRIN; VARGAS, 2002; BALESTRIN; VERSCHORE, 2008) e das contingências relacionadas aos objetivos comuns, tais como: necessidade, reciprocidade, eficiência e legitimidade (OLIVER, 1990; ALBANO; DE ARAUJO; REINHARD, 2017); c) Confiança: construída desde o período de formação da rede e desenvolvida ao longo do tempo, sendo suficiente para desenvolver relações de cumplicidade e eliminar a concorrência entre os órgãos públicos (COSTA, 2000; LAGEMANN, 2004); e d) Cooperação: estabelecimento de áreas de atuação, análise conjunta dos problemas (AGRANOFF, 2006), soluções em comum, definição da contribuição dos parceiros, eliminação de sobreposição de objetivos e ações, e preservação da autonomia dos participantes da rede (BALESTRIN; VERSCHOORE, 2008; FLEURY; OUVERNEY, 2007).

2 - Base institucional: Composta por duas categorias: a) Suporte político: estratégias de compartilhamento decisório, inclusão de interesses plurais, construção de consensos, gestão das relações com atores externos e mobilização de recursos externos (FLEURY; OUVERNEY, 2007); e b) Suporte técnico-operacional: articulação e integração dos recursos e de competências, racionalidade sistêmica na alocação de recursos, flexibilidade operacional (MARCON; MOINET, 2001) e criação de elementos (informações, relatórios, estudos etc.) de suporte decisório (PECl; COSTA, 2002).

3 - Coordenação e procedimentos: Composta por duas categorias: a) Organização articuladora: que desempenhe papel de órgão articulador, ou seja, organização focal integradora de esforços, empenhada mais que os outros membros da rede no papel da coordenação e articulação, tendo a missão de geração de consensos mais ágeis (LOIOLA; MOURA, 1996; FIALHO, 2005; PECl; COSTA, 2002); e b) Instrumentos de coordenação: instrumentos para manutenção da coordenação das atividades, de forma a reduzir as incertezas (instrumentos de tomada de decisão, instrumentos contratuais e instrumentos estratégicos) (FLEURY; OUVERNEY, 2007; BALESTRIN; VERSCHOORE, 2008; COSTA, 2010).

4 - Conectividade: aspectos relacionados à interação entre os órgãos. Composta por uma categoria: a) Interação constante e duradoura: interação suficiente para estimular a troca de recursos, estabelecer relações de confiança e formar capital social (HAGE; HOLLINGSWORTH, 2000; TSAI, 2000; NAKANO, 2005).

Segue, na próxima seção, a descrição dos resultados da pesquisa.

\section{Resultados}

Para coleta dos dados nos diversos órgãos, foram entrevistados profissionais com os cargos identificados no Quadro 4.

\section{Quadro 4 - Relação dos entrevistados}

\begin{tabular}{|c|c|}
\hline ORGÃOS PÚBLICOS & CARGO \\
\hline \multirow{3}{*}{ ANS } & Diretor \\
\hline & Diretor \\
\hline & Coordenador regional \\
\hline \multirow{3}{*}{ PROCON } & Coordenador regional \\
\hline & Coordenador municipal \\
\hline & Coordenador municipal \\
\hline \multirow{4}{*}{ MINISTÉRIO PÚBLICO } & Promotor de justiça \\
\hline & Promotor de justiça \\
\hline & Procurador da república \\
\hline & Procurador da república \\
\hline \multirow{3}{*}{ DEFENSORIA PÚBLICA } & Defensor público \\
\hline & \begin{tabular}{|l} 
Defensor público \\
\end{tabular} \\
\hline & Defensor público \\
\hline \multirow{3}{*}{ PODER JUDICIÁRIO } & Juiz de direito \\
\hline & Desembargador \\
\hline & Desembargador \\
\hline
\end{tabular}

Fonte: Elaborado pelos autores. 
Adiante é apresentada, no Quadro 5, a análise das condições necessárias para a criação de uma rede no setor de saúde suplementar do estado do RS, tendo como base as dimensões analíticas apresentadas na seção 4 deste trabalho. $O$ detalhamento dos resultados obtidos em cada um dos órgãos públicos do RS pesquisados (ANS, PROCON, Ministério Público, Defensoria Pública e Judiciário) estão apresentados, posteriormente, no Quadro 5.

No Quadro 5 é apresentada uma síntese dos resultados da avaliação das categorias propostas, tanto em cada órgão como no total dos entrevistados. Cabe esclarecer que o resultado "SIM" significa que ocorreu a aceitação da categoria pela maioria dos entrevistados nas questões estimuladas, com a apresentação de justificativas consistentes. Já o resultado "NÃO" significa que a categoria não foi aceita pela maioria dos entrevistados nas questões estimuladas pelos seguintes motivos: a) o entrevistado não aceitou a categoria; e b) o entrevistado aceitou a categoria, mas apresentou uma justificativa inconsistente. Além disso, os resultados destacados com asterisco $\left(^{*}\right)$ representam categorias que foram mencionadas espontaneamente.

Observando-se os resultados apresentados no Quadro 5 é possível confrontá-los com a posição dos especialistas no tema deste estudo. Assim, a categoria receptividade foi aceita pela maioria dos entrevistados, o que está aderente com a posição de Peci e Costa (2002), que sugere que a receptividade é um aspecto relevante no processo de construção de redes para a execução de políticas públicas. Já a categoria Estímulo, apesar de ter a aceitação da maioria dos entrevistados, não constituiu uma posição unânime.

A categoria Confiança, apesar de aceita pela maioria dos entrevistados também não apresentou aceitação unânime. Esse fato parece não muito coerente, já que autores como Costa (2000) são enfáticos ao destacar que a eficácia da colaboração interorganizacional é fortemente dependente dos níveis de confiança entre os atores. Talvez essa posição dos entrevistados seja uma decorrência do parcial desconhecimento ou transparência entre eles.

A categoria Cooperação apresentou situação similar à anterior; aceita pela maioria, mas não de forma unânime. Esse resultado também chama atenção, uma vez que a literatura da área tem apontado que a cooperação é um dos principais elementos para a formação de qualquer aliança entre organizações (GULATI; WOHLGEZOGEN; ZHELYAZKOV, 2012; ALBANO: DE ARAUJO; REINHARD, 2017).

\section{Quadro 5 - Resumo dos resultados da avaliação das categorias propostas para a criação da rede}

\begin{tabular}{|c|c|c|c|c|c|c|c|}
\hline \multirow[b]{2}{*}{ Dimensões } & \multirow[b]{2}{*}{ Categorias } & \multicolumn{5}{|c|}{ Resultados nos Órgãos Pesquisados } & \multirow[b]{2}{*}{ Total } \\
\hline & & ANS & PROCON & $\begin{array}{l}\text { Ministério } \\
\text { Público }\end{array}$ & \begin{tabular}{|} 
Defensoria \\
Pública
\end{tabular} & Judiciário & \\
\hline \multirow{4}{*}{$\begin{array}{l}\text { Ambiente } \\
\text { Favorável }\end{array}$} & Receptividade & $\stackrel{*}{\operatorname{sim}}$ & $\begin{array}{c}* \\
\operatorname{sim}\end{array}$ & $\begin{array}{c}* \\
\operatorname{sim}\end{array}$ & $\begin{array}{c}* \\
\operatorname{sim}\end{array}$ & $\begin{array}{c}* \\
\operatorname{sim}\end{array}$ & sim \\
\hline & Estímulo & $\stackrel{*}{*}$ & $\operatorname{sim}$ & não & $\stackrel{*}{*}$ & $\operatorname{sim}$ & sim \\
\hline & Confiança & $\operatorname{sim}$ & não & $\stackrel{*}{*}$ & $\operatorname{sim}$ & não & sim \\
\hline & Cooperação & $\operatorname{sim}$ & não & não & $\begin{array}{c}* \\
\operatorname{sim}\end{array}$ & $\operatorname{sim}$ & $\operatorname{sim}$ \\
\hline \multirow{2}{*}{$\begin{array}{l}\text { Base } \\
\text { Institucional }\end{array}$} & Suporte político & $\operatorname{sim}$ & $\operatorname{sim}$ & não & $\operatorname{sim}$ & não & não \\
\hline & $\begin{array}{l}\text { Suporte técnico- } \\
\text { operacional }\end{array}$ & $\operatorname{sim}$ & não & $\operatorname{sim}$ & $\operatorname{sim}$ & $\operatorname{sim}$ & $\operatorname{sim}$ \\
\hline \multirow{2}{*}{$\begin{array}{l}\text { Coordenação e } \\
\text { Procedimentos }\end{array}$} & $\begin{array}{l}\text { Organização } \\
\text { articuladora }\end{array}$ & não & não & $\stackrel{*}{*}$ & $\operatorname{sim}$ & $\operatorname{sim}$ & não \\
\hline & \begin{tabular}{|l} 
Instrumentos de \\
coordenação
\end{tabular} & sim & sim & não & sim & $\begin{array}{c}* \\
\text { não } \\
\end{array}$ & $\operatorname{sim}$ \\
\hline Conectividade & $\begin{array}{l}\text { Interação constante } \\
\text { e duradoura }\end{array}$ & $\begin{array}{c}* \\
\operatorname{sim}\end{array}$ & sim & não & $\begin{array}{c}* \\
\operatorname{sim}\end{array}$ & não & $\operatorname{sim}$ \\
\hline
\end{tabular}

Fonte: Elaborado pelos autores. 
Em termos da categoria Suporte político, ela não foi aceita pela maioria dos entrevistados, o que causa certa estranheza, já que um processo de formação de rede pública requer bases de suporte institucional, sendo o suporte político uma delas (FLEURY; OUVERNEY, 2007). Talvez a questão do risco de eventual influência de políticas partidárias tenha sido um dos motivadores desse resultado.

Considerando o Suporte técnico-operacional, essa categoria foi aceita pela maioria dos entrevistados, excetuando-se um órgão, o PROCON. A aceitação era esperada em função de que os recursos para trocas (informações, habilidades, tempo etc.) seriam o capital da rede e um dos elementos capazes de promover a sua operacionalidade (MARCON; MOINET, 2001).

A categoria Organização articuladora (da rede) não foi aceita pela maioria dos entrevistados, o que contradiz a posição de especialistas no tema. Portanto, uma organização focal, que execute o papel integrador da rede, garantindo que o pacto assumido pelos órgãos, é o que deve prevalecer, e deve coordenar as outras organizações, contribuindo para manter a rede unida (LOIOLA; MOURA, 1996).

A categoria Instrumentos de coordenação foi aceita pela maioria dos entrevistados, excetuando-se dois órgãos. A aceitação está de acordo com a visão de experts, pois instituir regras de atuação e construir mecanismos de decisão coletivos são alguns dos desafios para a criação e manutenção das redes de políticas (FLEURY; OUVERNEY, 2007).

No que tange à categoria Interação constante e duradoura, a aceitação se mostrou similar à categoria anterior, o que concorda com os autores pesquisados. A interação nas redes deve ser suficiente para estimular a troca de recursos e estabelecer relações de cumplicidade (MARCON; MOINET, 2001).

A partir da análise dos resultados da pesquisa, podem-se inferir as recomendações e ajustes relacionados à proposta das condições necessárias para a criação de uma rede para a regulação do setor de saúde suplementar do estado do RS.

Além das condições anteriormente analisadas, se evidencia o surgimento de uma nova condição necessária: a necessidade de se realizar nos órgãos um alinhamento do conhecimento sobre as categorias propostas, conceitos e elementos relacionados à atuação em rede. Isto devido à elevada quantidade de respostas confirmando as categorias, porém com a apresentação de justificativas inconsistentes.

São apresentados, no Quadro 6 (a seguir), a síntese dos resultados obtidos em cada uma das instituições pesquisadas, por meio das quais foram gerados os resultados apresentados anteriormente no Quadro 5. Cabe ressaltar que, em alguns casos, quando questionados sobre as categorias propostas, os entrevistados justificaram suas posições de forma superficial ou com argumentos que não correspondiam com a categoria apresentada. Essas respostas, portanto, foram tratadas como "justificativas inconsistentes". 


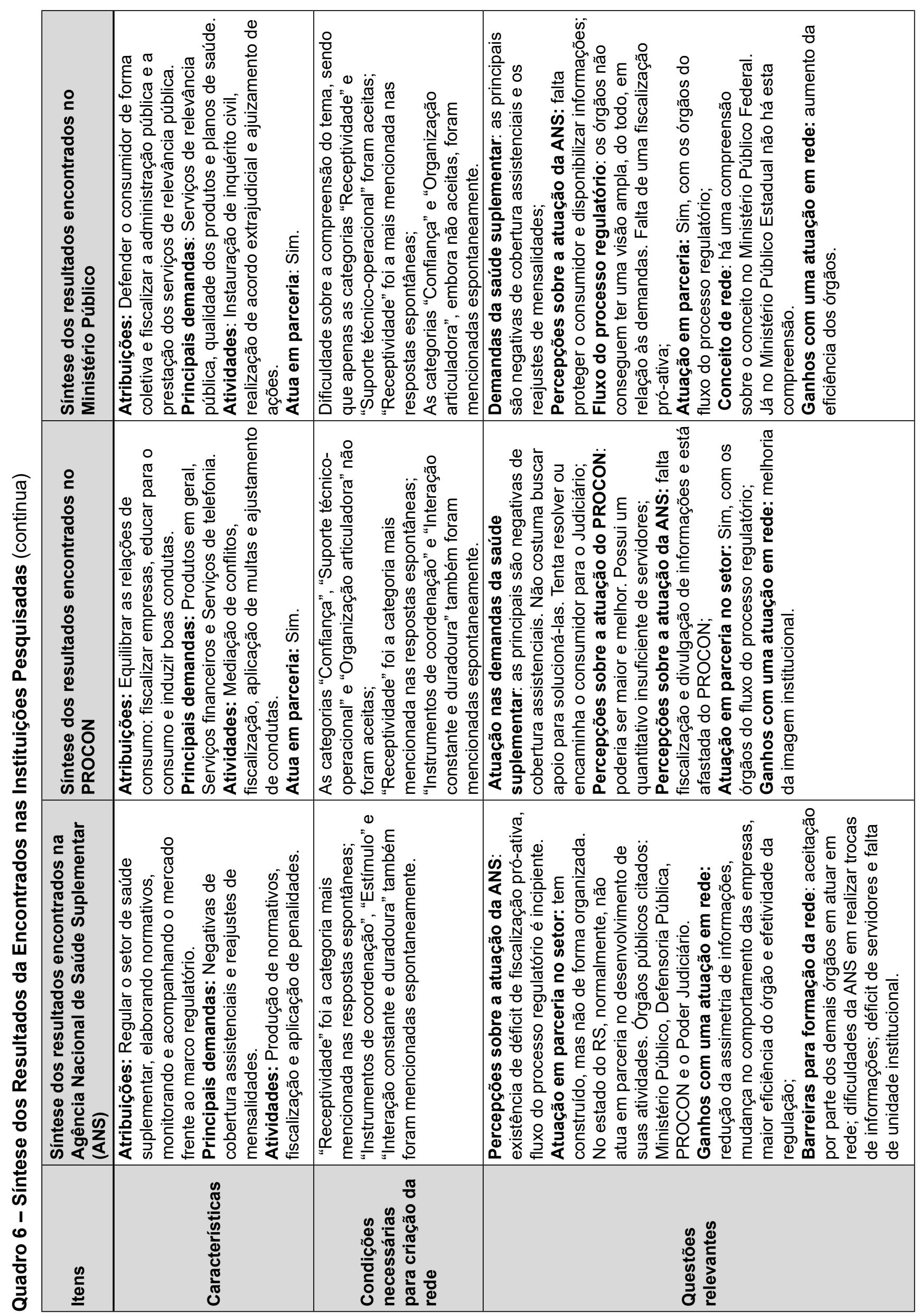




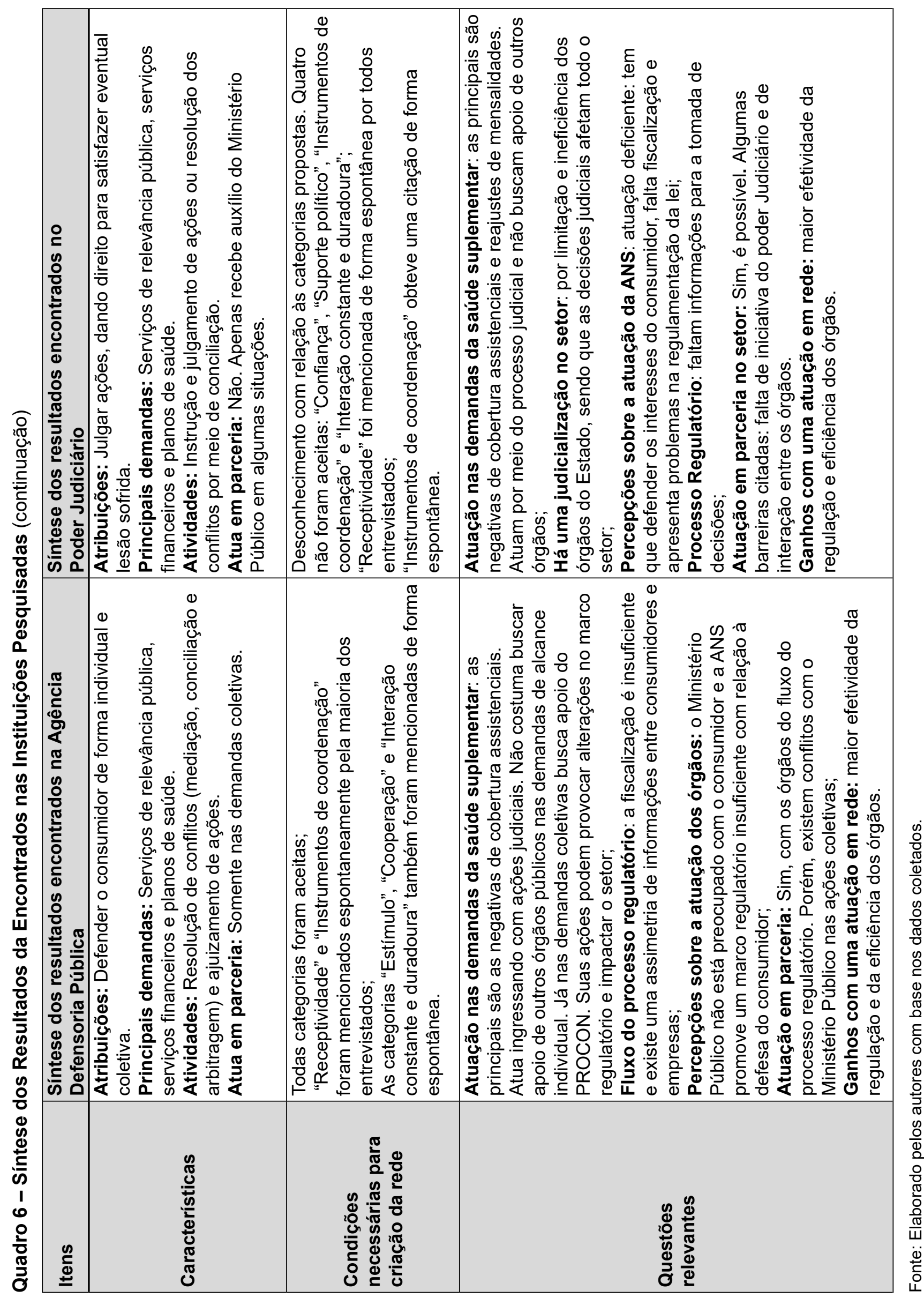


No Quadro 7 é apresentada uma síntese das condições necessárias para a criação de uma rede voltada a regulação da saúde suplementar do estado do RS, tendo em vista os ajustes e recomendações decorrentes da pesquisa de campo com os órgãos envolvidos.

\section{Quadro 7 - As condições necessárias para a criação de uma rede entre órgãos públicos no setor de saúde suplementar do Rio Grande do Sul / Brasil}

\begin{tabular}{|l|l|l|}
\hline \multicolumn{1}{|c|}{ Dimensões } & \multicolumn{1}{|c|}{ Categorias } & \multicolumn{1}{c|}{ Recomendações/ajustes } \\
\hline $\begin{array}{l}\text { Ambiente } \\
\text { favorável }\end{array}$ & $\begin{array}{l}\text { - Receptividade } \\
\text { - Estímulo } \\
\text { - Confiança } \\
\text { - Cooperação }\end{array}$ & $\begin{array}{l}\text { - Alinhar o conhecimento nos órgãos sobre as categorias estímulo, } \\
\text { confiança e cooperação; } \\
\text { - Compatibilizar as características dos órgãos (missões, valores, } \\
\text { objetivos particulares com objetivos da rede, processos e } \\
\text { procedimentos); } \\
\text { - Manter a autonomia dos órgãos dentro da rede, a fim de reduzir } \\
\text { conflitos prejudiciais a sua criação e manutenção. }\end{array}$ \\
\hline $\begin{array}{l}\text { Base } \\
\text { institucional }\end{array}$ & $\begin{array}{l}\text { - Suporte Político } \\
\text { - Suporte Técnico } \\
\text { Operacional }\end{array}$ & $\begin{array}{l}\text { As categorias representam etapas fundamentais dentro do } \\
\text { processo de institucionalização necessário à formação da rede; } \\
\text { Ministério Público e Judiciário. }\end{array}$ \\
\hline $\begin{array}{l}\text { Coordenação e } \\
\text { procedimentos }\end{array}$ & $\begin{array}{l}\text { - Organização } \\
\text { articuladora } \\
\text { - Instrumentos de } \\
\text { coordenação }\end{array}$ & $\begin{array}{l}\text { - Ajustar a categoria "Organização articuladora": articulação para } \\
\text { criação da rede deve ser realizada por um colegiado; } \\
\text { - Alinhar o conhecimento sobre a categoria "Instrumentos de } \\
\text { coordenação" no Ministério Público e Judiciário. }\end{array}$ \\
\hline Conectividade & $\begin{array}{l}\text { - Interação constante } \\
\text { e duradoura }\end{array}$ & $\begin{array}{l}\text { - Alinhar o conhecimento sobre esta categoria no Ministério Público } \\
\text { e Judiciário. }\end{array}$ \\
\hline
\end{tabular}

Fonte: Elaborado pelos autores.

Na próxima seção são apresentadas as considerações finais deste trabalho, as quais incluem implicações acadêmicas e gerenciais advindas do estudo realizado.

\section{Considerações Finais}

O presente estudo objetivou analisar as condições necessárias para a criação de uma rede no processo de regulação do setor de saúde suplementar no estado do RS no Brasil. Para tanto, foram levantadas as características dos órgãos públicos envolvidos no processo regulatório, que são: ANS, PROCON, Ministério Público, Defensoria Pública e Poder Judiciário. A partir do referencial teórico, foram propostas as condições básicas para a criação de uma rede, constituindo-se na estrutura de referência para a análise dos dados da pesquisa de campo.

Como principais necessidades identificadas no processo de regulação do mercado de saúde suplementar do estado do RS, destacam-se: a existência de um déficit de fiscalização (tanto reativa como pró-ativa); a existência de uma assimetria de informações entre consumidores e empresas; a falta de informações sobre a regulação do setor e para a tomada de decisões; e a falta de uma visão ampla, por parte dos órgãos envolvidos, em relação às demandas existentes e ao processo como um todo. Outra questão identificada foi a falta de busca por apoio dos órgãos públicos entre si para a resolução das demandas do setor.

Nesse sentido, também se destacam outras questões relevantes da pesquisa, tais como: as falhas da regulação da ANS, a superposição de atividades e atribuições dos órgãos, o desconhecimento dos órgãos sobre a atuação dos demais órgãos envolvidos, o desconhecimento de alguns gestores sobre os conceitos de rede e cooperação, os egos dos órgãos (não desejam organização articuladora), as críticas dos órgãos à atuação da ANS e o desconhecimento do fluxo da regulação, culminando com a falta de efetividade no processo.

A pesquisa permite concluir que os entrevistados acreditam ser possível a constituição de uma rede de órgãos atuando no processo de regulação de saúde suplementar. Integração que hoje não ocorre e que gera falta de maior efetividade nesse processo. Também se evidenciou a necessidade de se realizar nos órgãos 
um aprofundamento sobre o processo de regulação e um alinhamento do conhecimento sobre as categorias propostas, bem como sobre os conceitos e elementos relacionados à atuação em rede interorganizacional.

Devem ser observadas, no entanto, as limitações inerentes a esta pesquisa e suas eventuais implicações. Assim, o pequeno número de entrevistados, apesar da sua representatividade nos órgãos pesquisados, traz uma limitação natural à generalização dos resultados, quer nos órgãos envolvidos, no próprio setor, quer em outros estados do país. Além disso, a falta de visão sistêmica do processo de regulação como um todo por parte dos entrevistados oferece limitações à interpretação sobre os diferentes papéis dos órgãos e as possíveis vantagens de constituição de uma rede nesse processo e, por conseguinte, sobre as próprias conclusões alcançadas na pesquisa.

As implicações acadêmicas estão voltadas para a compreensão das relações existentes entre as categorias propostas, tais como a identificação de uma ordem de precedência, de dependência e/ou as relações de causa e efeito existentes entre elas.

Já as implicações gerenciais estão voltadas para o conhecimento, por parte dos gestores dos órgãos públicos, sobre o tema do presente estudo (redes interorganizacionais e cooperação). Assim, destaca-se a necessidade de se criar estruturas baseadas em relações de cooperação, em que esforços possam ser realizados para se chegar a entendimentos comuns e se definir um marco para uma ação rotineira. Outra implicação gerencial que se destaca é a definição das atribuições e o papel de cada órgão, de forma a complementar as ações dentro do processo regulatório do setor, haja vista que os diferentes órgãos envolvidos na rede resguardarão suas diversidades mesmo quando atuarem como parte de uma estrutura conjunta.

Uma reflexão final talvez deva ser apresentada. Parece ser relevante pesquisar e entender formas e procedimentos para "desenvolver uma atuação transversal para os diversos órgãos envolvidos nos processos públicos", oriundos de diferentes instâncias e poderes, no sentido de que a efetividade desses processos se sobreponha aos papéis e responsabilidades individuais dos encolvidos.

\section{Referências}

AGRANOFF, R. Inside Collaborative networks: ten lessons for public managers. Public Administration Review, [S.I.], v. 66, p. 56-65, Dec. 2006.

AGRANOFF, R.; MCGUIRE, M. Managing in network settings. Policy Studies Review, [S.I.], v. 16, n. 1, p. 18-42, 1999.

ALBANO, C. S.; ARAUJO, M. H. de; REINHARD, N. Fatores motivadores e facilitadores dos relacionamentos em redes: como os gestores públicos reconhecem esses fatores em dados governamentais abertos. Navus-Revista de Gestao e Tecnologia, Florianópolis, v. 7, n. 1, p. 73-92, 2017.

ALMEIDA, J. B. de. A proteção jurídica do consumidor. 7. ed. São Paulo: Saraiva, 2009.

AMANTINO-DE-ANDRADE, J. Redes de atores: uma nova forma de gestão das políticas públicas no Brasil? In: ENCONTRO NACIONAL DOS PROGRAMAS DE PÓS-GRADUAÇÃO EM ADMINISTRAÇÃO, 29., 2005, Salvador. Anais [...]. Salvador: ANPAD, 2005.

BAHIA, L. Planos privados de saúde: luzes e sombras no debate setorial dos anos 90 . Ciência \& Saúde Coletiva, Rio de Janeiro, v. 6, n.2, p.329-339, 2001.

BAHIA, L.; VIANA, A. L. Introdução. In: BRASIL. Regulação \& saúde: estrutura, evolução e perspectiva da assistência médica suplementar. Rio de Janeiro: Agência Nacional de Saúde Suplementar, 2002.

BALESTRIN, A.; VARGAS, M. L. Evidências Teóricas para a Compreensão das Redes Interorganizacionais. In: Encontro Nacional dos Programas de Pós-Graduação em Administração, 26, 2002. Recife. Anais [...]. Recife: Observatório da Realidade Organizacional: PROPAD/UFPE: ANPAD, 2002.

BALESTRIN, A.; VERSCHOORE, J. Redes de Cooperação Empresarial: estratégias de Gestão na Nova Economia. Porto Alegre: Artmed, 2008. 
BARBOSA, S. C. T.; MEDEIROS, J. J. Controle na implementação de programas públicos executados por redes interorganizacionais. In: Encontro Nacional dos Programas de Pós-Graduação em Administração, 26., 2002. Recife. Anais [...]. Recife: Observatório da Realidade Organizacional: PROPAD/UFPE: ANPAD, 2002.

BARDIN, L. Análise de Conteúdo. 3. ed. Lisboa: Edições 70, 2004.

BÖRZEL, T. Organizing babylon: on the different conceptions of policy networks. Public Administration, [S.I.], v. 76, p. 253-273, 1998.

RIO GRANDE DO SUL (Estado). Defensoria Pública. Relatório de Atividades. 2009. Disponível em: http://www.dpe.rs.gov.br/site/institucional_relatorio_anual.php. Acesso em: 18 out. 2010.

BRASIL. Lei 10.913, de 03 de janeiro de 1997. Institui o sistema estadual de defesa do consumidor do RS. Porto Alegre: Governo do Estado, 1997.

BRASIL. Lei 8.078, de 11 de setembro de 1990. [Código de Defesa do Consumidor]. Dispõe sobre a proteção do consumidor e dá outras providências. Diário Oficial da União, Brasília, 1990.

BRASIL. Ministério da Saúde. Agência Nacional de Saúde Suplementar. Instrução Normativa n. 1. Rio de Janeiro: ANS, 2006.

BRASIL. Ministério da Saúde. Agência Nacional de Saúde Suplementar. Caderno de Informação da Saúde Suplementar: beneficiários, operadoras e planos / Agência Nacional de Saúde Suplementar. Rio de Janeiro: Agência Nacional de Saúde Suplementar, 2010. Disponível em: http://www.ans.gov.br/index. php/materiais-para-pesquisas/perfil-do-setor/ publicacoes-ans Acesso em: 19 mar. 2011.

CARVALHO, E. B.; CECÍLIO, L. C. de O. A regulamentação do setor de saúde suplementar no Brasil: a reconstrução de uma história de disputas. Cad. de Saúde Pública, Rio de Janeiro, v. 23, n. 9, p. 21672177, 2007.

CASAROTTO, N.; PIRES, L. H. Redes de pequenas e médias empresas e desenvolvimento local. São Paulo: Atlas, 2001.

CASTELLS, M. A sociedade em rede. São Paulo: Paz e Terra, 1999a.

CASTELLS, M. Para o Estado-rede: globalização econômica e instituições políticas na era da informação. In: PEREIRA, L. C. B.; WILHEIM, J.; SOLA, L. (org). Sociedade e estado em transformação. São Paulo: UNESP, 1999b. p.147-171.

CECHIN, J. A história e os desafios da saúde suplementar: 10 anos de regulação. São Paulo: Saraiva: Letras \& Lucros, 2008.

CHEN, B. Antecedents or Processes? Determinants of perceived effectiveness of interorganizational collaborations for public service delivery. International Public Management Journal, [S.I.], v. 13, n. 4, p. 381-407, 2010.

COLLIS, J.; HUSSEY, R. Pesquisa em administração: um guia prático para alunos de graduação e pós-graduação. São Paulo: Bookman, 2005.

COSTA, A C. A confiança nas organizações: um imperativo nas práticas de gestão. In: RODRIGUES, S. B.; CUNHA, M. P. (org.) Estudos organizacionais: novas perspectivas na administração de empresas: uma coletânea luso-brasileira. São Paulo: Iglu, 2000. p. 284-305.

CRISÓSTOMO, S.; SANTOS, M. Participação pública na saúde: das ideias à ação em Portugal. Revista Crítica de Ciências Sociais, Coimbra, n. 117, p. 167-186, 2018.

CUNHA, P. C. M. A regulação jurídica da saúde suplementar no Brasil. Rio de Janeiro: Lumen Juris, 2003.

FIALHO, S. Metodologia para construção e gestão de redes de cooperação interorganizacionais. In: TEIXEIRA, F. (org.). Gestão de redes de Cooperação Interempresariais: em busca de novos espaços para o aprendizado e a inovação. Salvador: Casa da Qualidade, 2005. 
FIGUEIREDO, L. V. Curso de direito da saúde suplementar: manual jurídico de planos e seguros de saúde. São Paulo: MP Editora, 2006.

FLEURY, S. M.; OUVERNEY, M. A. Gestão de Redes: a estratégia de regionalização da política de saúde. Rio de Janeiro: Editora FGV, 2007.

FLEURY, S. Redes de políticas: novos desafios para a gestão pública. Administração em Dialógo, São Paulo, n. 7, p.77-89, 2005.

FLEURY, S.; MIGUELETTO, D.; BLOCH, R. Desafios da gestão de uma rede de políticas: o caso do Comitê das Entidades Públicas no Combate à fome e pela vida (COEP). In: ENCONTRO NACIONAL DOS PROGRAMAS DE PÓS-GRADUAÇÃO EM ADMINISTRAÇÃO, 26., 2002. Recife. Anais [...]. Recife: Observatório da Realidade Organizacional: PROPAD/UFPE: ANPAD, 2002.

GIOVANELLA, L.; RIBEIRO, J. M.; COSTA, N. R. Defesa dos consumidores e regulação dos planos de saúde. In: BRASIL. Regulação \& saúde: estrutura, evolução e perspectiva da assistência médica suplementar. Rio de Janeiro: Agência Nacional de Saúde Suplementar, 2002.

GOLDSMITH, S.; EGGERS, W. D. Governar em rede: o novo formato do setor público. Brasília: ENAP, 2006.

GRANDORI, A.; SODA, G. Inter-Firm networks: antecedents, mechanisms and forms. Organization Studies, [S.I.], v.16, n.2, p. 183-214, 1995.

GULATI, R.; WOHLGEZOGEN, F.; ZHELYAZKOV, P. The two facets of collaboration: Cooperation and coordination in strategic alliances. The Academy of Management Annals, [S.I.], v.6, n. 1, p. 531-583, 2012.

HAGE, J.; HOLLINGSWORTH, R. A strategy for the analysis of idea innovation networks and institutions. Organization Studies, [S.I.], v. 21, p. 971-1004, 2000.

KEAST, R.; MANDELL, M. P.; BROWN, K.; WOOLCOCK, G. Network Structures: Working Differently and Changing Expectations. Public Administration Review, [S.I.], v. 64, n. 3, p.363-371, 2004.

LAGEMANN, L. Fatores que influenciam a performance de redes de pequenas e médias empresas. 2004. Dissertação (Mestrado em Administração) - Universidade Federal do Rio Grande do Sul, Porto Alegre, 2004

LAZZARINI, M. A voz dos consumidores nas agências reguladoras. In: PROENÇA, J. D.; COSTA, P. V.; MONTAGNER, P. Desafios da regulação no Brasil. Brasília: ENAP, 2009. p.59-80.

LIMA, C. R. M.; LIMA, C. R. M. A regulação e a fiscalização do consumo de saúde suplementar no Brasil. Brasília: ANS, 2010. Disponível em: http://www.ans.gov.br/portal/site/biblioteca /trabalhos tecnicos05.asp. Acesso em: 15 jan. 2011.

LOIOLA, E.; MOURA, S. Análise das redes: uma contribuição aos estudos organizacionais. In: FISCHER, T. (org.) Gestão contemporânea: cidades estratégicas e organizações locais. Rio de Janeiro: FGV, 1996. p.53-68.

MACERA, A. P.; SAINTIVE, M. B. O mercado de saúde suplementar no Brasil. Brasília: Ministério da Fazenda, 2004.

MACIEL, C. O.; REINERT, M.; CAMARGO, C. Confiança e possibilidade de conflitos em redes estratégicas hierárquicas. Rev. Adm. Mackenzie, São Paulo, v. 12, n. 4, p. 182-208, 2011.

MARCON, C.; MOINET, N. Estratégia-Rede: ensaio de estratégia. Caxias do Sul: EDUCS, 2001.

MOURA, S. A construção de redes públicas na gestão local: algumas tendências recentes. Revista de Administração Contemporânea, Maringá, v. 2, n. 1, p. 67-85, 1998.

NAKANO, D. N. Fluxos de conhecimento em redes interorganizacionais: conceitos e fatores de influência. In: AMATO NETO, J. (org.). Redes entre organizações: domínio do conhecimento e da eficácia operacional. São Paulo: Atlas, 2005. p.54-67. 
NING, Y. Quantitative effects of drivers and barriers on networking strategies in public construction projects. International Journal of Project Management, [S.I.], v. 32, n.2, p. 286-297, Feb. 2013.

O'TOOLE, L. J. Treating networks seriously: practical and research-based agendas in public administration. Public Administrative Review, [S.I.], v. 57, n. 1, p. 45-53, 1997.

OLIVER, C. Determinants of interorganizational relationships: integration and future directions. Academy of Management Review, [S.I.], v. 15, n.2, p. 241-265, 1990.

OUVERNEY, A. L. Regionalização do SUS: uma análise da estratégia de integração intermunicipal. Administração em Diálogo, São Paulo, n. 7, p. 91-106, 2005.

$\mathrm{PECl}, \mathrm{A}$. Pensar e agir em rede: implicações na gestão das políticas públicas. In: ENCONTRO NACIONAL DOS PROGRAMAS DE PÓS-GRADUAÇÃO EM ADMINISTRAÇÃO, 24., 2000, Florianópolis. Anais [...]. Florianópolis: ENANPAD, 2000.

PECl, A.; COSTA, F. J. Redes para implementação de políticas públicas: Obstáculos e condições de funcionamento. In: ENCONTRO NACIONAL DOS PROGRAMAS DE PÓS-GRADUAÇÃO EM ADMINISTRAÇÃO, 26., 2002, Recife. Anais [...]. Recife: Observatório da Realidade Organizacional: PROPAD/UFPE: ANPAD, 2002

PETERS, D. T. J. M.; RAAB, J.; GREAUX, K. M.; STRONKS, K.; HARTING, J. Structural integration and performance of inter-sectoral public health-related policy networks: An analysis across policy phases. Health Policy, [S.I.], v. 121, n. 12, p. 1296-1302, 2017.

PROVAN, K. G.; LEMAIRE, R. H. Core concepts and key ideas for understanding public sector organizational networks: using research to inform scholarship and practice. Public Administration Review, [S.I.], v. 72, n. 5, p. 638-648, 2012.

ROESCH, S. M. A. Projetos de estágio e de pesquisa em administração: guia para estágios, trabalhos de conclusão, dissertações e estudos de caso. 3. ed. São Paulo: Atlas, 2009.

SANTOS, F. P. A regulação pública da saúde no Brasil: o caso da saúde suplementar. 2006. Tese (Doutorado em Ciências Médicas) - Programa de Pós-Graduação em Ciências Médicas, Universidade Estadual de Campinas, Campinas, 2006.

SOUZA, C. Políticas Públicas: uma revisão da literatura. Sociologias, Porto Alegre, v. 8, n. 16, p. 20-45, 2006.

TSAI, W. Social capital, strategic relatedness and the formation of interorganizational linkages. Strategic Management Journal, [S.I.], n. 21, p.925-939, 2000.

TURETA, C.; LIMA, J. B. de. Estratégia como prática social: o estrategizar em uma rede interorganizacional. Rev. Adm. Mackenzie, São Paulo, v. 12, n. 6, p. 76-108, 2011.

WACHHAUS, A. Networks in contemporary public administration: a discourse analysis. Administrative Theory \& Praxis, [S.I.], v. 31, n. 1, p. 59-77, 2009.

WALKER, R. M.; O'TOOLE, J. R. L. J.; MEIER, K. J. It's where you are that matters: the networking behaviour of english local government officers. Public Administration, [S.I.], v. 85, n. 3, p. 739-756, 2007.

Submetido em: 12/09/2016

Aceito em: 17/05/2019 